\title{
The analysis and diagnosis of machine tool spindle's fault based on the
}

\section{vibration test}

\author{
Bian Huamei, ${ }^{1, a}$, Li Zhaokun²,b \\ 1Beijing Vocational \& Technical Institute of Industry, Beijing,100042,China \\ ${ }^{2}$ Beijing Vocational \& Technical Institute of Industry, Beijing,100042,China \\ a13811096495@163.com,Tele:13811096495 \\ b2008lizhaokun@163.com,Tele:13671153498
}

Key word:CNC milling machine;Experimental analysis;Spindle system

Abstract:In order to accurately diagnose the fault type and location of CNC machine tool spindle, aimed at XKA714 CNC milling spindle's vibration characteristics, the article Uses the test analysis methods, Analyzed the Frequency characteristics of spindle system, and Achieved the vibration signal and vibration source of the spindle system, The Conclusion provide the basis for Machine maintenance.

\section{Introduction}

Numerical control machine tool is the common rotating machinery in manufacturing, Vibration is an important factor affecting the safe operation of the machine tool. It is the main cause of machining precision and cutting efficiency. Equipment failure often has a close relationship with vibration, Mechanical equipment in the work will inevitably occur to varying degrees of vibration. When the equipment is stable, the vibration has a typical extreme value and a certain characteristic. Once the parts inside the device have a defect, the size of the vibration and the type of vibration will change. When the vibration is serious, it will threaten the safe operation of the equipment. That the vibration signal can reflect the running state of the equipment. Because with the mechanical equipment in the operation of the vibrations it must be controlled in the range of permission. Due to the vibration caused by the operation of the mechanical equipment, the vibration will cause the deterioration of the equipment operation, and it must be controlled within the scope of the license. There are statistics show that equipment failure caused by vibration accounted for more than $60 \%$ in all kinds of faults.

Therefore, the vibration test and analysis of the equipment is an important means to achieve the equipment running status.

\section{Vibration test diagnosis technology}

Vibration test and diagnosis technology is an effective method of fault diagnosis based on vibration measurement.

Equipment vibration signal contains many important information in the system, parts and components, Such as wear, aging and fatigue caused by factors such as deterioration and failure, etc. We can monitor equipment running condition and fault type, fault source identification equipment. The vibration signal of the equipment is picked up and recorded by means of a variety of dynamic testing instruments, and it is analyzed and processed by the signal processing technology.

Vibration diagnosis technology is widely used in engineering practice because of the vibration 
signal measurement method is simple and the analysis theory is more mature.

Vibration test and analysis of CNC milling machine spindle

\section{Test and test system}

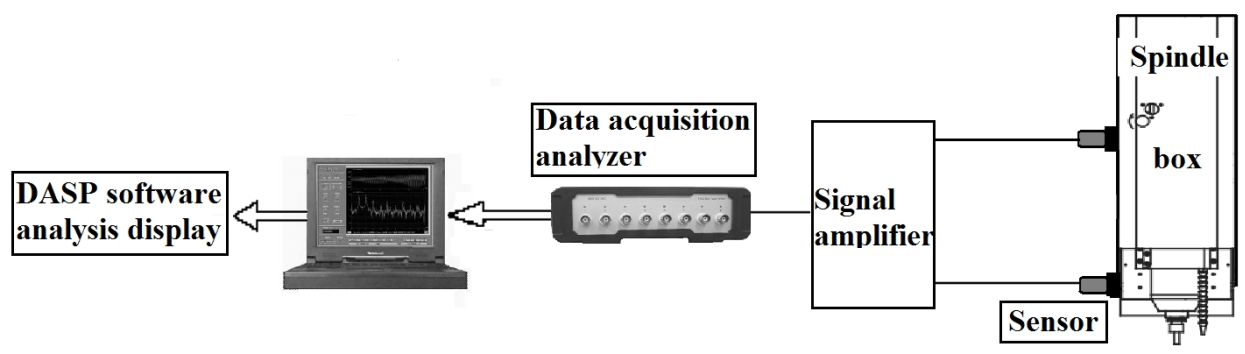

Fig. 1 Vibration measurement test system diagram

XKA714 CNC milling machine spindle as the research object in this paper, Test system composed of spindle, piezoelectric accelerometer, data acquisition and analysis system and the computer,As shown in Fig. 1.

The vibration signal is usually expressed by the displacement, velocity or acceleration of the structure, so it can be used in the measurement of three types of sensors. The frequency and amplitude range of different types of sensors are different. In this paper, the vibration characteristics of the spindle of CNC milling machine is measured by the piezoelectric acceleration sensor (9822A type, available frequency range $05 \mathrm{~Hz} \sim 5 \mathrm{KHz}$ ). Because the quality of acceleration sensor is small, When the sensor is glued to the structure, the influence on the structure is small. So the measurement is more accurate.

Data acquisition, analysis and processing system adopts four-channel parallel data acquisition processing system--INV3018A type high precision data acquisition and analysis software DASP analyzer (Data Acquisition \& Signal Processing) .The test system is developed and produced by China Orient Institute of Noise \& Vibration.

\section{Test and analysis program}

In the process of mechanical fault diagnosis, the most critical problem is signal feature extraction and state identification.

(1) Determination of spectral analysis

Frequency analysis is the most important and most commonly used method for signal processing in mechanical fault diagnosis.

The fault diagnosis information is extracted from the spectrum map (amplitude spectrum, phase spectrum, power spectrum, spectrum, spectrum and envelope spectrum, etc.) through Spectrum analysis of vibration signals. 


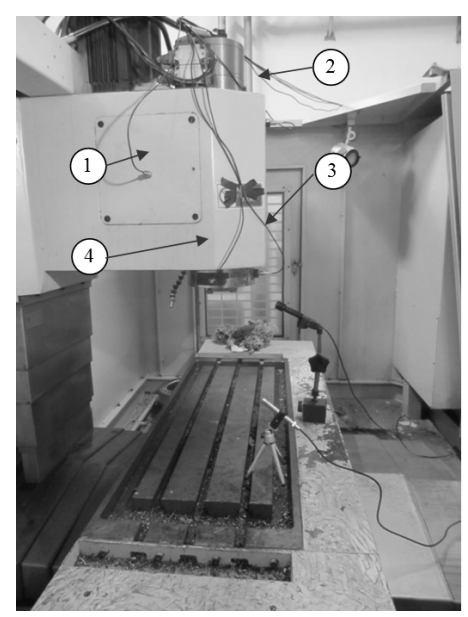

Fig. 2 Arrangement of measuring points of failure machine tools

(2) Selection of measuring points and arrangement of sensors

The measuring point selection of the vibration test test is based on the distribution position of the transmission parts in the main shaft structure of the machine tool. As shown in Fig. 2. The measuring point 1 cloth is arranged on the main shaft side wall, the measuring point 2 is arranged on the main shaft bearing radial direction, and the measuring point 3 and 4 cloth are arranged in the radial direction of the bearing under the main shaft, and the distance is 90 degrees.

(3) Equipment commissioning and sample setting

Sample set before calibration need to adjust the amplifier according to the vibration signal of the size of the gain in DASP software to adjust the appropriate range. Sampling frequency is 1024K. Each channel engineering unit is seted $\mathrm{m} / \mathrm{ss}$ (acceleration), At the same time, the vibration signal plus exponential window, to reduce leakage error.

(4) Test scheme determination

The test conditions are as follows: The machine condition for cutting state, When the feed rate is $200 \mathrm{~mm} / \mathrm{min}$ and the cutting depth is $1 \mathrm{~mm}$, the vibration signals of four directions are measured on spindle speed 300r/min, 500r/min, 700r/min, 900r/min and 1200r/min several speed。

\section{Analysis of failure mechanism of transmission parts}

\section{(1) Rolling bearing spectrum}

Specific diagnosis of bearing failure and bearing the specific parts of the damage, We need analyz frequency domain of the signal.

(1)Low frequency spectrum(Below $1 \mathrm{KHZ}$ ). Including the fault characteristic frequency of the bearing and the vibration characteristic frequency caused by the machining and assembling error. By analyzing the spectrum of the low frequency band, the corresponding bearing fault can be monitored and diagnosed. But because this frequency band is easy to be affected by other parts and structure in the machinery, and at the beginning of the failure to reflect the characteristic frequency of fault location, its energy is very small, often submerged in noise.

(2) Intermediate frequency spectrum $(1 \mathrm{kHz}-20 \mathrm{kHz})$. The natural vibration frequency of bearing elements caused by surface damage of bearing elements is mainly included. Analysis of the vibration signal in this frequency band can be used to diagnose the local damage of the bearing.

(3) High frequency spectrum(Above $20 \mathrm{kHz}$ ). If the acceleration sensor resonant frequency is higher $(40 \mathrm{kHz})$, then the impact caused by the bearing damage in the high frequency of $20 \mathrm{kHz}$ also has the energy distribution, the measured signal contains more than $20 \mathrm{kHz}$ high frequency components. The high frequency band signal can be analyzed and the corresponding bearing fault can be diagnosed. 


\section{(2) Gear failure mechanism}

The meshing vibration is caused by the continuous impact of the gears in the meshing process between the teeth and the teeth, and the frequency of the gear is equal to the forced vibration of the meshing frequency. This is the main cause of vibration of a gear meshing, the tooth elastic stiffness changes periodically, causing a collision in the tooth meshing gear caused by import when the frequency is equal to the vibration and noise of meshing frequency.

\section{Test result analysis}

(1) Bearing fault characteristic frequency calculation

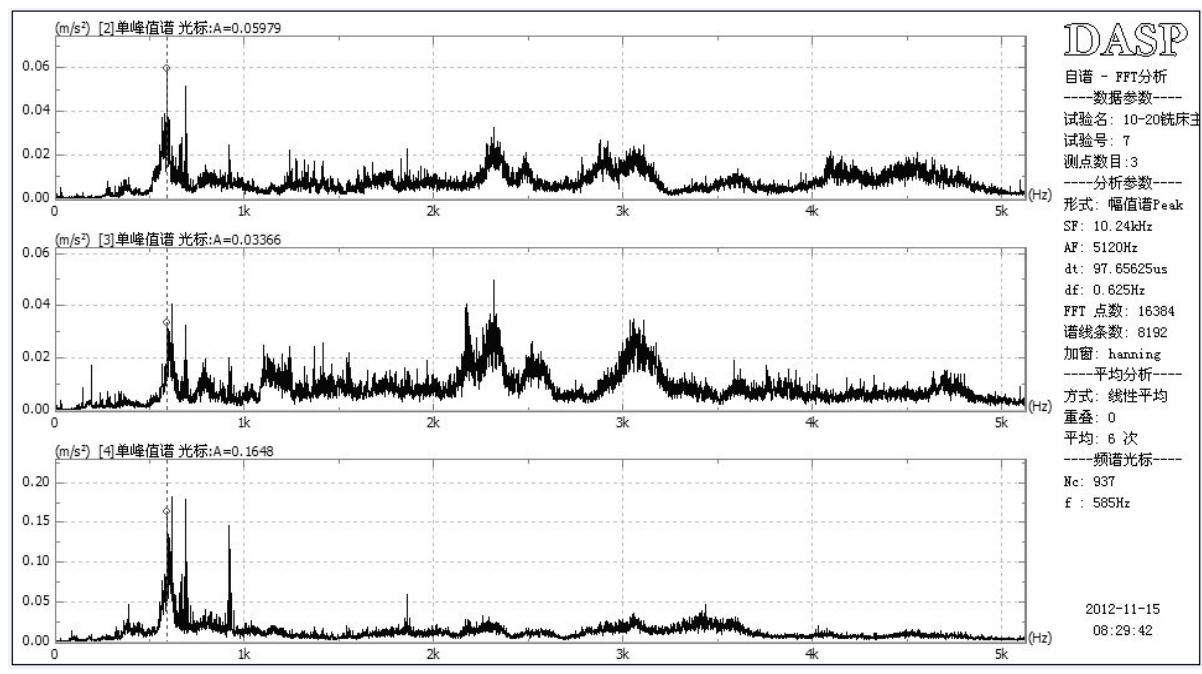

Fig. 3 Measuring point 2 4 (bearing) 300r/min spectrum

Fig. 3 for a certain speed under the spectrum of the bearing. The fault characteristic frequency of the bearing at different rotational speed as shown in Table 1,There is no obvious characteristic frequency of bearing fault by observing the frequency spectrum of a measuring point. Therefore, it is considered that there is no fault of bearing.

Table 1 Fault characteristic frequency of bearing at different speed

\begin{tabular}{ccccc}
\hline \multicolumn{5}{c}{ Upper bearing $(7211)$} \\
Speed (rpm) & Inner ring $(\mathrm{Hz})$ & Outer ring $(\mathrm{Hz})$ & Rolling body $(\mathrm{Hz})$ & Holder $(\mathrm{Hz})$ \\
300 & 32.2 & 42.8 & 13.3 & 2.85 \\
500 & 53.7 & 71.2 & 22.1 & 4.7 \\
700 & 75.2 & 99.7 & 31.0 & 6.6 \\
900 & 96.7 & 128.2 & 39.8 & 8.5 \\
1200 & 128.9 & 171.0 & 53.1 & 11.4 \\
& \multicolumn{5}{c}{ Lower bearing $(7014)$} \\
Speed (rpm) & Inner ring (Hz) & Outer ring $(\mathrm{Hz})$ & Rolling body $(\mathrm{Hz})$ & Holder $(\mathrm{Hz})$ \\
300 & 56.77 & 43.23 & 17.95 & 2.16 \\
500 & 94.6 & 72.0 & 29.9 & 3.6 \\
700 & 132.4 & 100.8 & 41.8 & 5.0 \\
900 & 170.3 & 129.6 & 53.8 & 6.4 \\
1200 & 227.0 & 172.9 & 71.8 & 8.6 \\
\hline
\end{tabular}

(2) Gear failure analysis

The vibration spectrum of the measuring point 1 as shown in Fig. 4. The zoom spectrum of the $700-1100 \mathrm{~Hz}$ frequency spectrum can be seen that the spectral line spacing is about $11 \mathrm{~Hz}$, The results indicate that there is obvious modulation phenomenon in the vibration signal, the first order 
frequency of the envelope spectrum is $11.25 \mathrm{~Hz}$. Through the gear parameter calculation shows that the two line frequency of the first order interval and envelope spectrum is the frequency of the intermediate shaft, can draw the middle level small gear is one of the source fault.

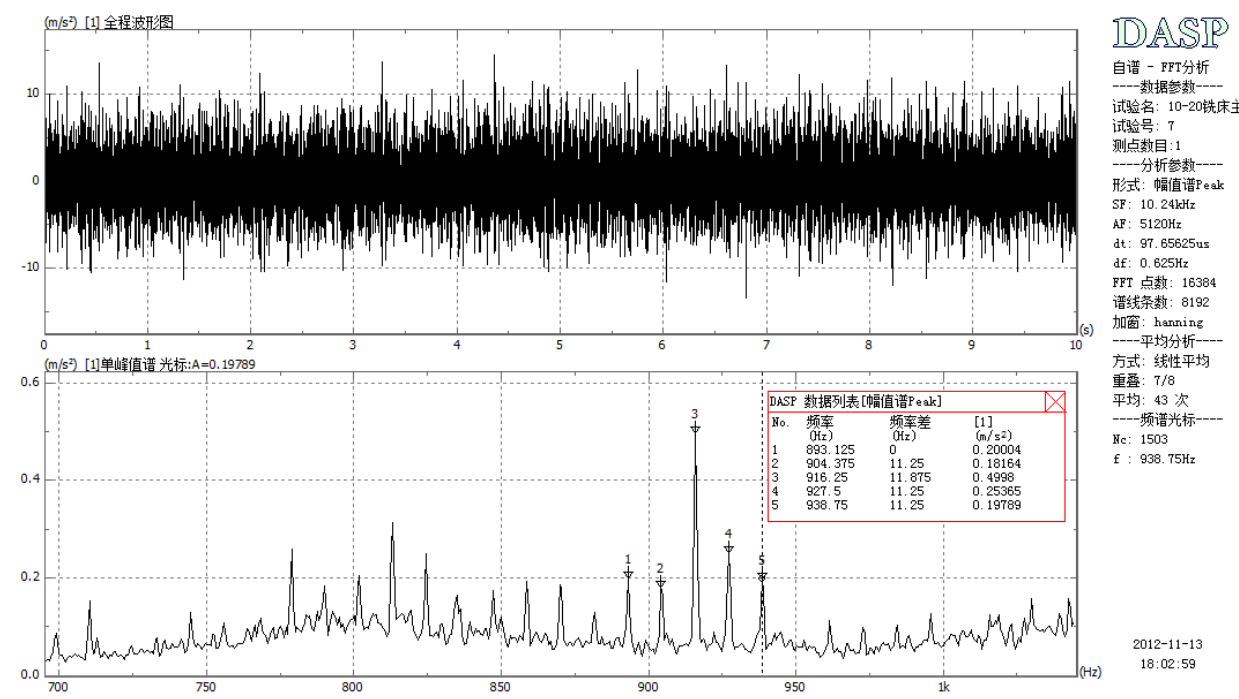

Fig. 4 300r/min time gear position spectrum

\section{Conclusion}

(1) Using the signal of the same type of normal operating machine tool as the datum, it can identify the fault location and type of the machine tool by comparing the vibration signal of the same position with the same type of the fault machine tool.

(2) With XKA714 CNC vertical milling machine spindle as the research object, The vibration test of the main shaft bearing and gear is carried out. The results of the test are analyzed in time domain and frequency spectrum, and the fault identification is carried out. analysis to get the position of machine tool failure and parts. Laid the foundation for fault diagnosis, provides guidance for the machine maintenance.

\section{Acknowledgment}

This research was supported by Beijing areal Project of Ministry of Education(KM2016-10853003),Collegestudents' scientific research innovation(bgzykey201615)and Beijing Polytechnic College in 2016(bgzykey201615,bgzykyt201617).

\section{Reference}

[1]Deng Xiaoyun. Research on the application of vibration diagnosis technology in the condition monitoring and fault diagnosis of CNC machine tools.[D]. Master of Engineering in Dalian Jiaotong University. 12.(2009).

[2] Bian Huamei. Experimental Modal Analysis of CNC Engraving Machine Spindle System.[J]. Coal Mine Machinery,41-42, 6.(2013) .

[3]Ni Zhenhua. Mechanics of vibration [M]. Xi'an Jiao Tong University press,(1989).

[4] Qu Weide, Tang Hengling editor. Handbook of mechanical vibration.[M]. China Machine Press. 5. (2000).

[5]Wang Xinqing. Study on diagnosis technology of gear box without disassembly.[D]. Doctoral Dissertation of Tianjin University. 2.(1998). 